\title{
Análise faunística de gafanhotos na Floresta Nacional de Chapecó, Santa Catarina
}

\author{
Cladis Juliana Lutinski ${ }^{1 *}$, Junir Antonio Lutinski ${ }^{1}$, Maria Katia Matiotti da Costa², Flávio Roberto Mello Garcia ${ }^{3}$ \\ ${ }^{1}$ Universidade Comunitária da Região de Chapecó (Unochapecó), PPG em Ciências Ambientais, CP 747, CEP 89809-000, Chapecó, SC, Brasil, \\ lutinski@gmail.com*, lutinski@ibest.com.br; ${ }^{2}$ Pontifícia Universidade Católica do Rio Grande do Sul (PUCRS), Faculdade de Biociências, Av \\ Ipiranga, 6681, Prédio 12 D, sala 45, CEP 90619-900, Porto Alegre, RS, Brasil, katiamatiotti@yahoo.com.br; ${ }^{3}$ Universidade Federal de Pelotas \\ (UFPel), Instituto de Biologia, Departamento de Zoologia e Genética, Laboratório de Ecologia de Insetos,CP 354, CEP 96010-900, Pelotas, \\ RS, Brasil, flavio.garcia@pq.cnpq.br
}

\begin{abstract}
Resumo - Realizou-se a análise faunística de gafanhotos coletados em três constituições vegetais da Flona de Chapecó. Coletas semanais foram realizadas em áreas de eucalipto, mata nativa e pinus durante o período de dezembro de 2003 a dezembro de 2004. Utilizaram-se armadilhas do tipo "pitfall", rede de varredura, guardachuva entomológico e "malaise". A fauna encontrada nas diferentes áreas foi caracterizada por meio dos índices de abundância, constância, dominância e frequência. Dezoito espécies foram comuns para as três áreas. As espécies Staurorhectus logicornis logicornis, Cylindrotettix sp. e Ommexecha virens ocorreram apenas em eucaliptos e pínus, enquanto, Scotussa lemniscata e Zoniopoda tarsata ocorreram para eucaliptos e mata nativa enquanto Amblytropidia sp. ocorreu somente em mata nativa e pínus. Tridactylus politus ocorreu somente em pínus. As espécies Allotruxalis gracilis, Dichroplus elongatus, Dichroplus misionensis e Ronderosia bergi foram muito frequentes, dominantes e muito abundantes na área de eucaliptos. A. gracilis, Metaleptea adspersa e D. misionensis foram muito frequentes, dominantes e muito abundantes na área de mata nativa. Metaleptea adspersa e $R$. bergi foram muito frequentes, dominantes e muito abundantes na área de pínus. Observou-se uma semelhança na diversidade de espécies entre as áreas, com maior abundância para a área de eucaliptos.
\end{abstract}

Termos para indexação: Ecologia, diversidade, Acridinae, desenvolvimento sustentável.

\section{Faunistic analyses of grasshoppers in the National Forest of Chapecó, Santa Catarina, Brazil}

\begin{abstract}
A study of the grasshopper fauna was performed through samples collected in three vegetal types in the National Forest of Chapecó. Weekly collections were carried out from December 2003 to December 2004. Pitfall traps, sweep nets, entomological umbrellas and malaise traps were used. Collections were repeated in areas with eucalyptus trees, native species and pine trees. The fauna found in different areas was classified by its abundance, constancy, dominance and frequency. Eighteen species were common in the three areas. Staurorhectus longicornis longicornis, Cylindrotettix sp. and Ommexecha virens were found only in eucalyptus and pine areas. Scotussa lemniscata and Zoniopoda tarsata were found in areas with eucalyptus and native trees. Amblytropidia sp. was found only in native vegetation and pines areas. Tridactylus politus was found just in the pine area. Allotruxalis gracilis, Dichroplus elongatus, Dichroplus misionensis and Ronderosia bergi were very frequent, dominant and very abundant in the eucalyptus tree area. A. gracilis, M. adspersa and D. misionensis were very frequent, dominant and very abundant in the native tree area. Metaleptea adspers $a$ and $R$. bergi were very frequent, dominant and very abundant in the pine area. A similarity in diversity of species was verified with greater abundance in the eucalyptus area.
\end{abstract}

Index terms: Ecology, diversity, Acridinae, sustainable development.

\section{Introdução}

Com o emergente interesse pelas questões ambientais tornou-se evidente a necessidade de um conhecimento mais amplo sobre a biodiversidade, bem como, da biologia e da ecologia das espécies. A partir destes estudos, torna-se possível a identificação de espécies ou grupos de espécies capazes de fornecer informações sobre a integridade e os processos que estão ocorrendo nos ambientes em que são encontrados (Lutinski \& Garcia, 2005). Neste contexto, as análises faunísticas permitem uma melhor caracterização das comunidades 
de insetos de um determinado ecossistema (Silveira Neto et al., 1995).

Os ortópteros datam nos registros fósseis a aproximadamente 300 milhões de anos. Atualmente, registram-se cerca de 20 mil espécies distribuídas em 28 famílias, sendo Acridoidea a superfamília mais numerosa, com cerca 10 mil espécies (Meyer, 2005). São conhecidos mundialmente e de importância econômica relevante devido aos danos econômicos causados em áreas agrícolas por algumas espécies. Além disso, são componentes fundamentais na cadeia alimentar e na alimentação de alguns povos (Costa-Neto, 2000).

Os gafanhotos representam uma ordem muito diversa de espécies que ocupam diferentes habitat (Kirk \& Bomar, 2005). Porém, a maioria vive nas regiões tropicais. Muitos vivem de modo solitário e outros vivem em grupos formando as "nuvens de gafanhotos", como são popularmente conhecidas na agricultura (Garcia, 2008). No Brasil, existem cerca 100 espécies de ortópteros, destas, menos de 23 exercem importância econômica (Centre for Overseas Pest Research, 1982; Lecoq, 1991).

Estudos sobre a ocorrência de Orthoptera registraram algumas espécies na cultura do milho no Estado de Santa Catarina (Pagliosa et al. 2001; Garcia et al. 2004; Carbonell et al., 2006). Schistocerca cancellata (Serville) e Schistocerca flavofasciata (De Geer) foram relatados na região oeste do estado em culturas de trigo (Silva et al., 1968), e Dichroplus misionensis (Acrididae) e Xyleus discoideus discoideus (Serville) (Romaleidae) como primeiro registro alimentando-se de plantas de laranjeira (Citrus sinensis Osbeck), no Município de Maravilha, SC (Campos et al., 2001). Também foi registrada a presença de 16 espécies em vegetação nativa no Município de Chapecó, SC (Graciani et al., 2005).

A Floresta Nacional de Chapecó (Flona) é uma área de manejo e conservação e possui fragmentos com características distintas (IBAMA, 2003) que possibilitam supor a existência de comunidades diferentes. Pelo deficiente conhecimento da ortopterofauna da região, justificou-se a realização desta pesquisa.

Objetivando conhecer o potencial da biodiversidade de gafanhotos existentes na região, desenvolveu-se a análise faunística de gafanhotos de três constituições vegetais da Floresta Nacional de Chapecó.

Pesq. flor. bras., Colombo, v. 31, n. 65, p. 43-50, jan./mar. 2011

\section{Material e métodos}

O estudo foi realizado na Gleba I da Floresta Nacional de Chapecó (Flona) localizada no Município de Guatambú, Santa Catarina, na localidade da Fazenda Zandavalli $\left(27^{\circ} 05^{\prime} 50^{\prime} \mathrm{S}\right.$; 52 $\left.52^{\circ} 46^{\prime} 40^{\prime \prime} \mathrm{W}\right)$, em três áreas, com cerca de cinco hectares cada uma. A área 1 tem como vegetação predominante uma plantação de eucalipto (Eucalyptus saligna Smith e Eucalyptus grandis Hill) com três anos de idade. A área 2 é coberta por mata com araucária (Floresta Ombrófila Mista) e Floresta Estacional Decidual. A área 3 está coberta por uma plantação de pínus (Pinus taeda Linnaeus e Pinus elliottii Engelm) com 45 anos de idade (Ibama, 2003).

As amostragens foram semanais no período de dezembro de 2003 a dezembro de 2004. Nas coletas foram utilizadas armadilhas do tipo ativo, caso da rede de varredura e guarda-chuva entomológico e as armadilhas passivas "pitfall" e "malaise".

O esforço amostral empregado por coleta, igualmente em cada área, consistiu em 20 minutos de varredura para rede de varredura em aproximadamente 60 sacudidelas em um total de 10 arbustos para o guardachuva entomológico; em 10 armadilhas tipo "pitfall" distribuídas a intervalos de $10 \mathrm{~m}$ entre si em transectos lineares perpendiculares da borda para o interior; e uma armadilha do tipo "malaise" instalada na região central de cada uma das áreas.

Os espécimes coletados foram acondicionados em frascos com álcool $70 \%$ e transportados ao laboratório de Entomologia da Unochapecó, onde foram triados e identificados. A confirmação dos táxons foi realizada com base na classificação proposta por Rowell (2001).

A caracterização de cada comunidade através da medida faunística da constância para cada espécie coletada foi determinada pela equação $\mathrm{C}=(\mathrm{p} \times 100) / \mathrm{N}$ (Silveira Neto et al., 1976):

$\mathrm{C}=$ constância em percentual;

$\mathrm{p}=\mathrm{n}^{\mathrm{o}}$ de coletas contendo a espécie em estudo;

$\mathrm{N}=\mathrm{n}^{\circ}$ total de coletas efetuadas.

Assim, as espécies foram classificadas em constantes, acessórias ou acidentais.

Os valores foram obtidos a partir dos cálculos dos intervalos de confiança de $1 \%$ e $5 \%$ sobre as médias obtidas dos números de espécimes totais de cada espécie, em cada área. Foram obtidos intervalos em torno da 
média usados para classificar as espécies em raras, dispersas, comuns, abundantes e muito abundantes.

A dominância das espécies em cada comunidade foi determinada pelo cálculo do limite de dominância calculado a partir da equação LD = (1 / S) x100 citada Sakagami \& Laroca (1971).

LD = limite de dominância;

$\mathrm{S}=$ número total de espécies.

Através deste parâmetro, as espécies foram classificadas em dominantes, quando os valores da frequência apresentaram-se superiores a este limite e não dominantes, quando os valores encontrados foram menores.

A dominância das espécies de gafanhotos foi avaliada através do índice de equitabilidade (J') proposto por Pinto-Coelho (2000), em que o índice de equitabilidade varia entre zero e um, sendo o resultado maior que 0,5 considerado indicativo de uniformidade na distribuição das espécies no local avaliado.

$\mathrm{J}^{\prime}=\mathrm{H}^{\prime} /(\ln (\mathrm{S}))$ onde:

$\mathrm{J}$ ' = índice de eqüitabilidade;

H' = índice de diversidade de Shannon e Weaner (1949);

$\mathrm{S}=$ número total de espécies.

As espécies foram, de acordo com o cálculo da frequência, agrupadas em pouco frequente, frequente ou muito frequente (Thomazini \& Thomazini, 2002). Este parâmetro faunístico foi determinado através da ocorrência de cada espécie em relação ao total de coletas combinadas com os métodos de captura utilizados no decorrer do estudo (Silveira Neto et al., 1976) e os valores obtidos a partir dos cálculos dos intervalos de confiança de 5\% sobre as médias obtidas dos registros totais para cada espécie, em cada área.

A diversidade em cada área foi mensurada através dos índices de Shannon \& Weaner, proposto por PintoCoelho (2000). A análise faunística foi realizada a partir do número de espécimes coletados de cada espécie, para cada área e método.

\section{Resultados e discussão}

Na Flona, foram amostrados um total de 25 espécies, 21 gêneros e cinco famílias (Tabela 1). Dos 2.325 espécimes coletados, $63,8 \%$ foram capturados na área de eucalipto, $25,3 \%$ na área de mata nativa e $10,8 \%$ na área de pínus. Os índices de diversidade e equitabilidade foram 2,7 e 0,$86 ; 2,5$ e 0,$82 ; 2,6$ e 0,84 , respectivamente (Tabela 2).
Tabela 1. Número total de gafanhotos capturados em cada área das três formações florísticas da Flona de Chapecó, SC, no período de dezembro de 2003 a dezembro de 2004.

\begin{tabular}{|c|c|c|c|c|}
\hline \multirow[b]{2}{*}{ Subfamílias } & \multirow[b]{2}{*}{ Espécies } & \multicolumn{3}{|c|}{ Áreas de estudo } \\
\hline & & 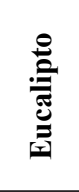 & 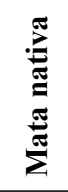 & $\stackrel{气}{E}$ \\
\hline \multirow[t]{3}{*}{ Acridinae } & A. gracilis & 152 & 62 & 30 \\
\hline & M. adspersa & 80 & 83 & 40 \\
\hline & P. graminea & 29 & 10 & 7 \\
\hline Copiocerinae & A. lineatus & 3 & 4 & 2 \\
\hline \multirow[t]{6}{*}{ Gomphocerinae } & Amblytropidia sp. & 39 & 3 & 1 \\
\hline & O. punctata & 16 & 18 & 7 \\
\hline & P. nigromarginata & 82 & 31 & 7 \\
\hline & R. brunneri & 80 & 17 & 16 \\
\hline & R. pictus & 24 & 26 & 2 \\
\hline & $\begin{array}{l}\text { S. longicornis lon- } \\
\text { gicornis }\end{array}$ & 0 & 0 & 3 \\
\hline \multirow[t]{2}{*}{ Leptysminae } & C. chacoensis & 5 & 2 & 1 \\
\hline & Cylindrotettix sp. & 7 & 0 & 1 \\
\hline \multirow[t]{4}{*}{ Melanoplinae } & D. elongatus & 172 & 24 & 8 \\
\hline & D. misionensis & 210 & 128 & 18 \\
\hline & R. bergi & 130 & 74 & 35 \\
\hline & S. lemniscata & 7 & 1 & 0 \\
\hline Ommatolampinae & A. flavolineata & 30 & 11 & 13 \\
\hline \multirow[t]{5}{*}{ Romaleinae } & C. speciosa & 55 & 7 & 2 \\
\hline & $\begin{array}{l}\text { S. viridicata } \\
\text { viridicata }\end{array}$ & 21 & 34 & 3 \\
\hline & $\begin{array}{l}X . \text { discoideus } \\
\text { discoideus }\end{array}$ & 65 & 22 & 10 \\
\hline & Z. iheringi & 12 & 23 & 6 \\
\hline & Z. tarsata & 3 & 1 & 0 \\
\hline Ommexechinae & O. virens & 180 & 0 & 9 \\
\hline Tetriginae & T. subulata & 82 & 8 & 30 \\
\hline \multirow[t]{2}{*}{ Tridactylinae } & T. politus & 0 & 0 & 1 \\
\hline & Total & 1484 & 589 & 252 \\
\hline
\end{tabular}

O índice de diversidade é a relação entre o número de espécies e o número de indivíduos de uma comunidade (Silveira Neto et al., 1976). Este índice permitiu a comparação entre as diferentes comunidades. Entretanto, não se observou diferença significativa na diversidade das áreas avaliadas, corroborando os resultados obtidos por Andersen et al. (2001), na Austrália. Esses valores parecem estar relacionados ao fato de o grupo ser muito diverso e ocupar numerosos habitats (Kirk \& Bomar, 2005). 
Já os índices de equitabilidade mostraram-se proporcionais aos índices de diversidade, indicando um padrão similar na distribuição das espécies nas áreas. Neste parâmetro, ambas as áreas avaliadas apresentaram índices acima de 0,5 e com proximidade entre si. Este parâmetro é o segundo componente mais importante na diversidade de espécie (Odum, 2001). Sendo assim, quanto maior este valor, maior será a diversidade de espécie, que no presente trabalho apresentou correlação positiva significativa para todas as áreas (Tabela 2).

Tabela 2. Análise faunística das espécies de gafanhotos capturados em três formações florísticas da Flona de Chapecó, SC, no período de dezembro de 2003 a dezembro de 2004.

\begin{tabular}{|c|c|c|c|c|c|c|c|c|c|c|c|c|}
\hline \multirow{3}{*}{ Espécies } & \multicolumn{12}{|c|}{ Áreas de coleta/índices } \\
\hline & \multicolumn{4}{|c|}{ Eucalipto } & \multicolumn{4}{|c|}{ Mata nativa } & \multicolumn{4}{|c|}{ Pínus } \\
\hline & $\mathbf{F}$ & D & $\mathbf{A}$ & $\mathbf{C}$ & $\mathbf{F}$ & D & $\mathbf{A}$ & $\mathbf{C}$ & $\mathbf{F}$ & D & $\mathbf{A}$ & $\mathbf{C}$ \\
\hline A. gracilis & $\mathrm{k}$ & $\mathrm{s}$ & $\mathrm{m}$ & $\mathrm{w}$ & $\mathrm{k}$ & $\mathrm{s}$ & $\mathrm{m}$ & $\mathrm{y}$ & $\mathrm{k}$ & $\mathrm{s}$ & $\mathrm{m}$ & $\mathrm{z}$ \\
\hline M. adspersa & $\mathrm{f}$ & $\mathrm{s}$ & $\mathrm{c}$ & $\mathrm{w}$ & $\mathrm{k}$ & $\mathrm{s}$ & $\mathrm{m}$ & $\mathrm{y}$ & $\mathrm{k}$ & $\mathrm{s}$ & $\mathrm{m}$ & $\mathrm{y}$ \\
\hline P. graminea & $\mathrm{p}$ & $\mathrm{n}$ & $\mathrm{r}$ & z & $\mathrm{p}$ & $\mathrm{n}$ & $\mathrm{r}$ & z & $\mathrm{p}$ & $\mathrm{n}$ & $\mathrm{d}$ & $\mathrm{z}$ \\
\hline A. lineatus & $\mathrm{p}$ & $\mathrm{n}$ & $\mathrm{r}$ & $\mathrm{z}$ & $\mathrm{p}$ & $\mathrm{n}$ & $\mathrm{r}$ & $\mathrm{z}$ & $\mathrm{p}$ & $\mathrm{n}$ & $\mathrm{r}$ & $\mathrm{z}$ \\
\hline Amblytropidia sp. & 0 & 0 & 0 & 0 & $\mathrm{p}$ & $\mathrm{n}$ & $\mathrm{r}$ & $\mathrm{z}$ & $\mathrm{p}$ & $\mathrm{n}$ & $\mathrm{r}$ & $\mathrm{z}$ \\
\hline O. punctata & $\mathrm{p}$ & $\mathrm{n}$ & $\mathrm{r}$ & $\mathrm{y}$ & $\mathrm{P}$ & $\mathrm{n}$ & d & $\mathrm{z}$ & $\mathrm{p}$ & $\mathrm{n}$ & $\mathrm{d}$ & $\mathrm{z}$ \\
\hline P. nigromarginata & $\mathrm{p}$ & $\mathrm{n}$ & $\mathrm{r}$ & $\mathrm{z}$ & $\mathrm{f}$ & $\mathrm{s}$ & $\mathrm{c}$ & $\mathrm{z}$ & $\mathrm{p}$ & $\mathrm{n}$ & $\mathrm{d}$ & $\mathrm{z}$ \\
\hline R. brunneri & $\mathrm{k}$ & $\mathrm{s}$ & a & $\mathrm{y}$ & $\mathrm{p}$ & $\mathrm{n}$ & $d$ & z & $\mathrm{k}$ & $\mathrm{s}$ & $\mathrm{m}$ & $\mathrm{Z}$ \\
\hline R. pictus & $\mathrm{f}$ & $\mathrm{s}$ & $\mathrm{c}$ & $\mathrm{y}$ & $\mathrm{f}$ & $\mathrm{n}$ & $\mathrm{c}$ & $\mathrm{y}$ & $\mathrm{p}$ & $\mathrm{n}$ & $\mathrm{r}$ & $\mathrm{z}$ \\
\hline S. longicornis longicornis & $\mathrm{p}$ & $\mathrm{n}$ & $\mathrm{r}$ & $\mathrm{z}$ & 0 & 0 & 0 & 0 & $\mathrm{p}$ & $\mathrm{n}$ & $\mathrm{r}$ & $\mathrm{z}$ \\
\hline C. chacoensis & $\mathrm{p}$ & $\mathrm{n}$ & $\mathrm{r}$ & z & $\mathrm{p}$ & $\mathrm{n}$ & $\mathrm{r}$ & $\mathrm{z}$ & $\mathrm{p}$ & $\mathrm{n}$ & $\mathrm{r}$ & $\mathrm{z}$ \\
\hline Cylindrotettix sp. & $\mathrm{p}$ & $\mathrm{n}$ & $\mathrm{r}$ & $\mathrm{z}$ & 0 & 0 & 0 & 0 & $\mathrm{p}$ & $\mathrm{n}$ & $\mathrm{r}$ & $\mathrm{z}$ \\
\hline D. elongatus & $\mathrm{k}$ & $\mathrm{s}$ & $\mathrm{m}$ & $\mathrm{w}$ & $\mathrm{f}$ & $\mathrm{n}$ & $\mathrm{c}$ & z & $\mathrm{f}$ & $\mathrm{n}$ & $\mathrm{c}$ & $\mathrm{z}$ \\
\hline D. misionensis & $\mathrm{k}$ & $\mathrm{s}$ & $\mathrm{m}$ & $\mathrm{w}$ & $\mathrm{k}$ & $\mathrm{s}$ & $\mathrm{m}$ & $\mathrm{y}$ & $\mathrm{k}$ & $\mathrm{s}$ & $\mathrm{m}$ & $\mathrm{z}$ \\
\hline R. bergi & $\mathrm{k}$ & $\mathrm{s}$ & $\mathrm{m}$ & $\mathrm{w}$ & $\mathrm{k}$ & $\mathrm{s}$ & $\mathrm{m}$ & $\mathrm{z}$ & $\mathrm{k}$ & $\mathrm{s}$ & $\mathrm{m}$ & $\mathrm{y}$ \\
\hline S. lemniscata & $\mathrm{p}$ & $\mathrm{n}$ & $\mathrm{r}$ & $\mathrm{z}$ & $\mathrm{p}$ & $\mathrm{n}$ & $\mathrm{r}$ & $\mathrm{z}$ & 0 & 0 & 0 & 0 \\
\hline A. flavolineata & $\mathrm{p}$ & $\mathrm{n}$ & $\mathrm{r}$ & z & $\mathrm{p}$ & $\mathrm{n}$ & $\mathrm{r}$ & z & $\mathrm{f}$ & $\mathrm{s}$ & $\mathrm{c}$ & $\mathrm{z}$ \\
\hline C. speciosa & $\mathrm{f}$ & $\mathrm{n}$ & $\mathrm{c}$ & $\mathrm{z}$ & $\mathrm{p}$ & $\mathrm{n}$ & $\mathrm{r}$ & $\mathrm{z}$ & $\mathrm{p}$ & $\mathrm{n}$ & $\mathrm{r}$ & $\mathrm{z}$ \\
\hline S. viridicata viridicata & $\mathrm{p}$ & $\mathrm{n}$ & $\mathrm{r}$ & z & $\mathrm{f}$ & $\mathrm{s}$ & $\mathrm{c}$ & $\mathrm{y}$ & $\mathrm{p}$ & $\mathrm{n}$ & $\mathrm{r}$ & $\mathrm{z}$ \\
\hline$X$. discoideus discoideus & $\mathrm{f}$ & $\mathrm{s}$ & $\mathrm{c}$ & $\mathrm{y}$ & $\mathrm{f}$ & $\mathrm{n}$ & $\mathrm{c}$ & z & $\mathrm{f}$ & $\mathrm{n}$ & $\mathrm{c}$ & $\mathrm{z}$ \\
\hline Z. iheringi & $\mathrm{p}$ & $\mathrm{n}$ & $\mathrm{r}$ & z & $\mathrm{f}$ & $\mathrm{n}$ & $\mathrm{c}$ & z & $\mathrm{p}$ & $\mathrm{n}$ & $\mathrm{r}$ & $\mathrm{z}$ \\
\hline Z. tarsata & $\mathrm{p}$ & $\mathrm{n}$ & $\mathrm{r}$ & $\mathrm{z}$ & $\mathrm{p}$ & $\mathrm{n}$ & $\mathrm{r}$ & $\mathrm{z}$ & 0 & 0 & 0 & 0 \\
\hline O. virens & $\mathrm{k}$ & $\mathrm{s}$ & $\mathrm{m}$ & $\mathrm{y}$ & 0 & 0 & 0 & 0 & $\mathrm{f}$ & $\mathrm{n}$ & $\mathrm{c}$ & $\mathrm{z}$ \\
\hline T. subulata & $\mathrm{k}$ & $\mathrm{s}$ & a & $\mathrm{y}$ & $\mathrm{p}$ & $\mathrm{n}$ & $\mathrm{r}$ & $\mathrm{z}$ & $\mathrm{k}$ & $\mathrm{s}$ & $\mathrm{m}$ & $\mathrm{z}$ \\
\hline T. politus & 0 & 0 & 0 & 0 & 0 & 0 & 0 & 0 & $\mathrm{p}$ & $\mathrm{n}$ & $\mathrm{r}$ & $\mathrm{z}$ \\
\hline Índice de Diversidade & \multicolumn{4}{|c|}{2,68} & \multicolumn{4}{|c|}{2,51} & \multicolumn{4}{|c|}{2,64} \\
\hline Equitabilidade & \multicolumn{4}{|c|}{0,86} & \multicolumn{4}{|c|}{0,82} & \multicolumn{4}{|c|}{0,84} \\
\hline
\end{tabular}




\section{Área de eucalipto}

$\mathrm{Na}$ área de eucalipto foram encontradas nove subfamílias, destas, Gomphocerinae e Romaleinae apresentaram cinco espécies cada, Acridinae apresentou três e Leptysminae duas espécies. O maior número de espécimes foi encontrado para a subfamília Melanoplina, tendo sido registrado 210 espécimes para $D$. misionensis e 172 para $D$. elongatus, 130 para $R$. bergi e sete para $S$. lemniscata. Uma espécie foi amostrada para cada uma das subfamílias Ommatolampinae, Ommexechinae, Tetriginae e Copiocerinae (Tabela 1). Foi constatado que Eutropidacris cristata (L., 1758) (Orthoptera: Acrididae) ataca árvores adultas de eucalipto em Lavras, Minas Gerais (Zanetti et al., 2003), o que pode ocorrer também com algumas das espécies amostradas.

As espécies Allotruxalis gracilis (Giglio-Tos), Dichroplus elongatus, D. misionensis e $R$. bergi foram muito frequentes, dominantes, muito abundantes e constantes. Já $O$. virens foi muito frequente, dominante e muito abundante, e as espécies Rhammatocerus brunneri (Giglio-Tos) e Tetrix subulata (Linaeus) foram muito frequentes e dominantes. As espécies M. adspersa (Rehn) e Rhammatocerus pictus (Bruner) foram frequentes, dominantes e comuns. A espécie Chromacris speciosa (Thumberg) foi comum e frequente, entretanto, não foi dominante. Todas as demais espécies ocorreram de forma rara ou dispersas, acidentais ou acessórias, não dominantes e pouco frequentes nesta área (Tabela 2).

\section{Área de mata nativa}

No ambiente nativo foram encontradas oito subfamílias, entre elas, Gomphocerinae e Romaleinae apresentaram cinco espécies cada. O maior número de espécimes foi encontrado para a subfamília Melanoplinae, tendo sido registrados 128 espécimes para $D$. misionensis e 24 para $D$. elongatus, 74 para $R$. bergi e um para S. lemniscata. A subfamília Acridinae apresentou três espécies, enquanto Ommatolampinae, Leptysminae, Tetriginae e Copiocerinae apenas uma espécie cada (Tabela 1).

Nesta área, nenhuma espécie foi constante. As espécies A. gracilis, M. adspersa e D. misionensis foram muito frequentes, dominantes, muito abundantes e acessórias. A espécie $R$. bergi foi muito frequente, dominante, muito abundante, porém acidental (Tabela 2).

As espécies Peruvia nigromarginata (Scudder) e Staleochlora viridicata viridicata (Serville) foram frequentes e dominantes. Enquanto $R$. pictus, D. elongatus, Xyleus discoideus discoideus (Serville) e Zoniopoda iheringi (Pictet \& Saussure) foram frequentes e não dominantes. Todas as demais foram pouco frequentes, não dominantes, comuns ou raras e acidentais (Tabela 2).

\section{Área de pínus}

A área de pinus destacou-se com dez subfamílias, destas, Gomphocerinae apresentou seis espécies, Romaleinae quatro, Acridinae e Melanoplinae três, Leptysminae duas. Já as subfamílias Ommatolampinae, Copiocerinae, Ommexechinae, Tetriginae e Tridactylinae apresentaram apenas uma espécie cada (Tabela 1). Para Tridactylinae, a espécie T. politus (Bruner) ocorreu com exclusividade nesta área.

Nesta área, nenhuma espécie foi constante. As espécies M. adspersa e $R$. bergi foram muito frequentes, dominantes, muito abundantes e acessórias. Já $A$. gracilis, $R$. brunneri, D. misionensis e T. subulata foram muito frequentes, dominantes e muito abundantes. Somente Abracris flavolineata (De Geer) foi frequente e dominante. D. elongatus, $X$. discoideus discoideus e $O$. virens foram frequentes. Todas as demais foram pouco frequentes, não dominantes, comuns ou raras e acidentais (Tabela 2).

\section{Influência do tipo de vegetação na ocorrência de gafanhotos}

Embora houvesse variação nas medidas faunísticas de algumas espécies em cada área, observou-se uniformidade na distribuição das subfamílias. Das dez subfamílias coletadas no estudo, Gomphocerinae, Romaleinae, Acridinae, Leptysminae, Melanoplinae Ommatolampinae, Tetriginae e Copiocerinae ocorreram nas três áreas. A subfamília Ommexechinae ocorreu somente nas áreas de pínus e eucalipto e Tridactylinae ocorreu somente na área de pínus (Tabela 1).

Gomphocerinae foi a subfamília que apresentou maior ocorrência de espécies em todas as áreas estudadas. Este resultado corrobora com Graciane et al. (2005), que obteve maior número de espécies para esta subfamília de Acrididae no estudo desenvolvido em um fragmento de floresta nativa. Conforme DeBrey et al. (1993), esta subfamília é formada por espécies muito ativas e com grande capacidade de dispersão.

A subfamília Romaleinae, representada por $C$. speciosa, S. viridicata viridicata, $X$. discoideus discoideus e $Z$. iheringi comuns para as três áreas e $Z$. tarsata comum para mata nativa e eucalipto, equipara-se aos resultados encontrados por Graciane at al. (2005), que obteve as mesmas espécies em um fragmento de

Pesq. flor. bras., Colombo, v. 31, n. 65, p. 43-50, jan./mar. 2011 
floresta nativa. Romaleinae apresenta ampla distribuição pela América do Sul (Capinera et al. 2001), com espécies potencialmente pragas que atacam grande diversidade de plantas silvestres e cultivadas (Duranton et al., 1987; Carbonell et al., 2006) caracterizando, assim, a uniformidade do grupo na área.

A subfamília Melanoplinae apresentou espécies com grande número de espécimes em todas as áreas. A grande quantidade de indivíduos coletados está relacionada à subfamília tida como pragas de grande potencial, com grande habilidade e capacidade de dispersão (DeBrey et al., 1993).

Silveira Neto et al. (1976) afirmaram que a abundância é o número de indivíduos por unidade de superfície, a qual varia no espaço de uma comunidade para outra e no tempo com as flutuações populacionais. Verificou-se variação na abundancia entre as comunidades das diferentes áreas, bem como entre as espécies. As espécies $A$. gracilis, $D$. misionensis e $R$. bergi foram muito abundantes nas três áreas. A abundância destas espécies pode ter sido facilitada pela diversidade de ambientes, propiciando as condições ideais para espécies generalistas. Conforme Carbonell et al. (2006), Allotruxalis gracilis é uma espécie associada a gramíneas e plantas cultivadas. A subfamília Melanoplinae agrupa espécies generalistas e potencialmente pragas, com habilidade de dispersão (DeBrey et al., 1993). Entretanto, a espécie R. bergi é generalista de ambientes (Carbonell et al., 2006) e $M$. adspersa prefere vegetações mais densas, corroborando o parâmetro muito abundante verificado nas áreas de mata nativa e pínus.

Rhammatocerus brunneri e T. subulata foram muito abundantes apenas para a área de pínus. A diversidade vegetal presente na regeneração desta área criou as condições adequadas na disponibilidade de alimentos e abrigo para estas espécies. A espécie T. subulata apresenta hábitos arborícolas (Riede, 1987), o que corrobora as condições físicas com ampla vegetação arbustiva, na referida área.

Dichroplus elongatus e O. virens foram muito abundantes na área de eucalipto, onde se verifica espaços abertos e vasta presença de gramíneas. Segundo Torrusio et al. (2002), D. elongatus habita diferentes ambientes, além disso, apresenta forte associação positiva com plantações (gramíneas, forrageiras, árvores frutíferas e leguminosas) e locais fortemente alterados, enquanto, O. virens, prefere ambientes abertos (Duranton et al., 1987), relacionando, assim, sua abundância com a área de eucalipto.
Xyleus discoideus discoideus foi comum para as três áreas, caracterizando-se generalista de ambientes e alimentação, também verificado em Carbonell et al. (2006) em que tal espécie alimenta-se dos mais variados vegetais e adapta-se a diferentes ambientes.

Das 23 espécies amostradas na área de eucalipto, sete foram abundantes ou muito abundantes e quatro foram comuns. A superioridade no número de espécimes e espécies observado na área de eucalipto atribuise às características físicas da vegetação jovem do reflorestamento, a qual propicia vasta presença de gramíneas e incidência de luz, criando condições favoráveis para o grupo. Conforme Mathur \& Soni (1983), plantações de eucalipto apresentam dossel mais aberto que permite maior penetração de radiação e de água de chuva, explicando assim a maior diversidade e população de plantas em seu sub-bosque. Além disso, a pobreza de inimigos naturais favorece a proliferação do grupo. O eucalipto é uma espécie exótica e cultivada como monocultura, que pode modificar o ambiente. Desta forma, são poucas as espécies no Brasil que estarão aptas para viver em plantio de eucalipto (Alcides \& Pereira, 2007).

Já nas áreas de mata nativa e pínus onde a diversidade vegetal é maior, o equilíbrio populacional do grupo é visível e a prevalência de espécies foi rara ou dispersa, observando-se poucas espécies de alta abundância. Tais dados concordam com os resultados de Graciane et al. (2005), onde a ocorrência para a maioria das espécies foi rara ou dispersa, com apenas a espécie Allotruxalis strigata (Bruner) ocorrendo de forma comum.

$\mathrm{O}$ equilíbrio observado na área da mata nativa atribui-se ao clímax ecológico e a presença de mata fechada e sombria. Já na área de pínus, a elevada regeneração vegetal possibilitou o estabelecimento equilibrado e diversificado de espécies. Conforme Lima (2007), as áreas de mata nativa e pínus na Flona de Chapecó apresentam características muito próximas de regeneração estrutural, proporcionando um ambiente arbóreo semelhante entre ambas.

Foi observado que muitas espécies predominam na Flona e não a dominam. Apenas A. gracilis, $M$. adspersa, $D$. misionensis e $R$. bergi foram dominantes em todas as áreas, corroborando o resultado de Graciane et al. (2005), em que $D$. misionensis e $M$. adspersa foram dominantes além de Amblytropidia sp. e Orphulella punctata (De Geer) em um fragmento florestal nativo. 
As espécies $A$. gracilis, D. misionensis e $R$. bergi foram muito frequentes em todas as áreas estudadas. Este resultado aponta espécies frequentes para a região, onde o único registro indicava apenas espécies pouco frequentes, conforme Graciane et al. (2005).

As espécies A. gracilis, M. adspersa, D. elongatus, $D$. misionensis e $R$. bergi foram constantes na área de eucalipto, confirmando o resultado obtido por Graciane et al. (2005), em que O. punctata e A. strigata (Bruner) foram constantes no Município de Chapecó.

\section{Métodos de captura}

A maioria dos gafanhotos $(82,6 \%)$ foi amostrada com pitfall e rede-de-varredura. $\mathrm{O}$ número de gafanhotos amostrados em cada um dos métodos foi variável de acordo com o tipo de ambiente. No eucalipto, a maioria dos espécimes foi capturada na armadilha de solo seguido pela rede-de-varredura; na área de pínus e de mata nativa, na rede-de-varredura (Figura 1), confirmando a rede-de-varredura como um bom método para captura destes insetos (Richman et al., 1993).

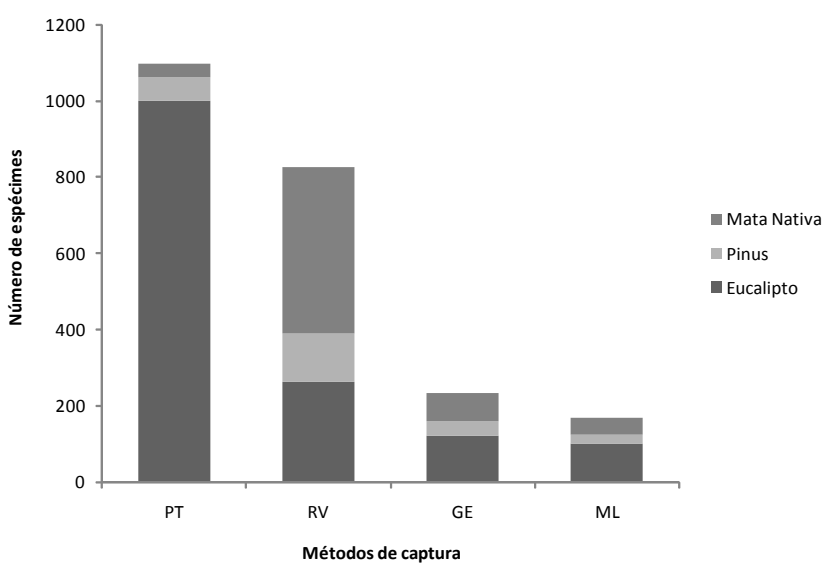

Figura 1. Número de espécimes de gafanhotos capturados semanalmente em áreas de eucalipto, mata nativa e pínus na Floresta Nacional de Chapecó, Guatambú, SC, no período de dezembro de 2003 a dezembro de 2004. Sendo: PT = pitfall, $\mathrm{RV}=$ rede-de-varredura, $\mathrm{GE}=$ guarda-chuva-entomológico e $\mathrm{ML}=$ Malaise.

\section{Conclusões}

Há semelhança na diversidade de espécies entre as áreas com maior abundância e constância para a área de eucalipto.

Os locais fortemente alterados podem favorecer a abundância de algumas espécies como D. misionensis $I$, D. elongatus e $R$. bergi.
As espécies $A$. gracilis, M. adspersa, D. misionensis e $R$. bergi foram predominantes em todas as áreas estudadas.

É importante a conjugação de diversos métodos de captura de gafanhotos em estudos faunísticos, mas sempre é necessária a utilização de armadilhas pitfall e rede-de-varredura.

\section{Agradecimentos}

Ao Fundo de Apoio à Pesquisa da Unochapecó, pela bolsa de Cladis J. Lutinski. Ao CNPq pela Bolsa de Produtividade em Pesquisa de Flávio R. M. Garcia.

\section{Referências}

ALCIDES, F. R.; PEREIRA, L. P. C. Considerações ecológicas sobre plantios de Eucalipto. In: VIII CONGRESSO DE ECOLOGIA DO BRASIL, 09., 2007, Caxambu - MG. Anais. SEB. Universidade Federal de Viscosa. 2007. Disponível em: $<$ http://www.seb-ecologia.org.br/viiiceb/pdf/1554.pdf $>$. Acesso em: 28 jun. 2009.

ANDERSEN, A. N.; LUDWIG; J. A.; LOWE, L. M.; RENTZ, D. C. F. Grasshopper biodiversity and bioindicators in Australian tropical savannas: Responses to disturbance in Kakadu National Park. Austral Ecology, Sydney, v. 26, n. 3, p. 213-222, jun. 2001.

CAMPOS, J. V.; GARCIA, F. R. M.; COSTA, M. K. M. Ocorrência de duas espécies de gafanhotos (Orthoptera, Caelifera) alimentandose de plantas cítricas no Extremo Oeste de Santa Catarina, Brasil. Biotemas, Florianópolis, v. 14, n. 2, p. 157-160, 2001.

CARBONELL, C. S.; CIGLIANO, M. M.; LANGE, C. E. Espécies de acridomorfos (Orthoptera) de Argentina y Uruguai/ Acridomorph (Orthoptera) species of Argentina and Uruguai. Argentina: The Orthopterists' Society at the Museu de La Plata, 2006. (CD ROM. Publications on Orthopteran Diversity).

CAPINERA, J. L.; SCHERER, C. W.; SQUITIER, J. M. Grasshoppers of Florida. Invertebrates of Florida series. Gainesville: University Press of Florida. 2001. 143 p. Disponível em: $<$ http://entnemdept.ufl.edu/ghopper/ghopper.html $>$. Acesso em: 30 jun. 2010.

CENTRE FOR OVERSEAS PEST RESEARCH (London, UK). The locust and grasshopper agricultural manual. London, 1982. 690p. il.

COSTA-NETO, E. Insetos no cardápio. Ciência Hoje, Brasília, v. 27, n. 161, p. 63-65. 2000.

DeBREY, L. D.; BREWER, M. J.; LOCKWOOD, J. A Rangeland Grasshopper Management. Wyoming: Agricultural Experiment Station. College of Agriculture. University of Wyoming. 1993. Disponível em: <http://www.uwyo.edu/ grasshoppersupport/html_pages/rgmanage.htm>. Acesso em: 30 jun. 2010. 
DURANTON, J. F.; LAUNOIS, M.; LUANG, H. L.; LECOQ, M. Guia Práctico de Luta contra os Gafanhotos Devastadores no Brazil. Roma. FAO-CIRAD-PRIFAS. 1987. 343 p.

GARCIA, F. R. M.; NARDI, N.; COSTA, M. K. M.; BRESCOVIT A. D. Ocorrência de artrópodes em lavoura de milho (Zea mays) no município de Arvoredo, SC. Bioikos, Campinas, v. 18, n. 1, p. 21-28, jan./jun. 2004.

GARCIA, F. R. M. Zoologia agrícola: manejo ecológico de pragas. 3. ed. Porto Alegre: Ed. Rígel, 2008. 256 p.

GRACIANI, C.; GARCIA, F. R. M.; COSTA, M. K. M. Análise faunística de gafanhotos (Orthoptera, Acridoidea) em fragmento florestal próximo ao Rio Uruguai, município de Chapecó, SC. Biotemas, Florianópolis, v. 18, n. 2, p. 89-87. 2005.

IBAMA. Floresta Nacional de Chapecó. O meio ambiente em sintonia com a conservação. Chapecó, 2003. 217 p.

KIRK, K.; BOMAR, C. B. Guide to the Grasshoppers of Wisconsin. Madison, Bureau of Integrated Science Services, Wisconsin Department of Natural Resources, 2005. 154 p.

LECOQ, M. Gafanhotos do Brasil: natureza do problema e bibliografia. Montpellier: CIRAD-PRIFAS; Campinas: EmbrapaNMA, 1991. 157 p. broch., il., A5

LIMA, R. F. Regeneração natural das espécies arbóreas no sub-bosque dos povoamentos nativos e de Pinus taeda l. da FLONA de Chapecó, Guatambu-SC. 2007. 79f. Dissertação (Mestre em Ciências Agrárias) - UNOCHAPECO, Chapecó.

LUTINSKI, J. A.; GARCIA, F. R. M. Análise faunística de Formicidae (Hymenoptera: Apocrita) em ecossistema degradado no município de Chapecó, Santa Catarina. Biotemas, Florianópolis, v. 2, n. 18, p. 73-86, 2005.

MATHUR, H. N.; SONI, P. Comparative account of undergrowth under Eucalyptus and Sal in three different localities of Doon Valley. Indian Forest, v. 109, n. 12, p. 882-890, 1983.

MEYER, J. R. Orthoptera. The orthopterists' Society. North Carolina, USA. State University, 2005. Disponível em: <http:// www.cals.ncsu.edu/course/ent425/compendium/orthop.html $>$. Acesso em: 30 jun. 2010.

ODUM, E. P. Fundamentos de Ecologia. 6. ed. Lisboa: Fundação Calouste Gulbenkian, 2001. 927 p.

PAGLIOSA, G.; GARCIA, F. R. M.; CHAGAS, E. T. G.; MUSSIOL, I. Z.; GASPARETO, A.; SALDAVEGO, M. M. Artrópodes ocorrentes em lavoura de milho (Zea mays) em Chapecó, SC In: REUNIAO TECNICA CATARINENSE DE MILHO E FEIJAO, 3., 2001, Chapeco, SC. Resumos... Chapeco: EPAGRI/CPPP, 2001. p. 120-124.
PINTO-COELHO, R. M. Fundamentos em ecologia. Porto Alegre: Artmed, 2000. 252 p.

RICHMAN, D. B.; LIGHTFOOT, D. C.; SUTHERLAND, C. A.; FERGUSON, D. J. A manual of the grasshoppers of New Mexico Orthoptera: Acrididae and Romaleidae. New Mexico State University Cooperative Extension Service las Cruces, NM. Set. 1993. Disponível em: <http://www.sidney.ars.usda.gov/ grasshopper/extrnlpg/ghwywest/ghnmtoc.htm>. Acesso em: 10 jan. 2007.

RIEDE, K. A Comparative study of mating behaviour in some Neotropical Grasshoppers (Acridoidea). Ethology, Berlim, v. 76, p. 265-296, 1987.

ROWELL, H. Acridoidea. 2001. Version 01 January 2001 (temporary). Disponível em: <http://tolweb.org/Acridoidea/13290 /2001.01.01 inTheTreeofLifeWeb>. Acesso em 30 jun. 2010.

SAKAGAMI, S. F.; LAROCA, S. Relative abundance, phenology and flower visits of apid bees in Eastern Paraná, South Brazil (Hym., Apidae). Kontyü, Tokyo, v. 39, p. 213-30, 1971.

SILVA, A. G. d'A. e.; GONÇALVES, C. R.; GALVÃO, D. M.; GONÇALVES, A. J. L.; GOMES, J.; SILVA, M. N.; SIMONI, L. Quarto catálogo de insetos que vivem nas plantas do Brasil: seus parasitas e predadores. Rio de Janeiro: Ministério da Agricultura, Laboratório Central de Patologia Vegetal, 1968, t. 1, p. 2., 622 p.

SILVEIRA NETO, S.; NAKANO, O.; BARBIN D.; VILLA NOVA, N. A. Manual de Ecologia dos Insetos. Sao Paulo: Agronômica Ceres, 1976. 419 p.

SILVEIRA NETO, S.; MONTEIRO, R. C.; ZUCCHI, R. A.; MORAES, R. C. B. Uso da análise faunística de insetos na avaliação de impacto ambiental. Scientia Agricola, Piracicaba, v. 52, n.1, p. 9-15, jan./abr.1995.

THOMAZINI, M. J.; THOMAZINI, P. Diversidade de abelhas (Hymenoptera: Apoidea) em inflorescências de Piper hisoidinervum (C.D.C.). Neotropical Entomology. Londrina, v. 31, n. 1, p 27-34. Mar.2002. Disponível em: <http://www.scielo. br/scielo.php?script=sci_arttext\&pid=S1519-566X20020001000 $04 \& \operatorname{lng}=$ pt\&nrm $=$ iso $\&$ tlng $=$ pt $>$. Acesso em: 20 Jun. 2009. doi: 10.1590/S1519-566X2002000100004.

TORRUSIO, S.; CIGLIANO, M. M.; DE WISIECKI, M. L. Grasshopper (Orthoptera: Acridoidea) and plants community relationships in the argentine Pampas. Journal of biogeography, Oxford, v. 29, p. 221-229, mar. 2002.

ZANETTI, R.; SOUZA-SILVA, A.; MOURA, M. A.;

ZANUNCIO, J. C. Ocorrência do gafanhoto-do-coqueiro

Eutropidacris cristata (Orthoptera: Acrididae) atacando plantas de eucalipto em Minas Gerais. Revista Árvore, Viçosa, v. 27, n.1, p. 105-107, jan./fev.2003.

Recebido em 09 de novembro de 2009 e aprovado em 28 de dezembro de 2010 